Canadian

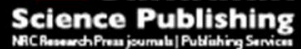

Canadian Journal of Physiology and Pharmacology Revue canadienne de physiologie et pharmacologie

\title{
Short-term effects of hemodiafiltration versus conventional hemodialysis on erythrocyte performance
}

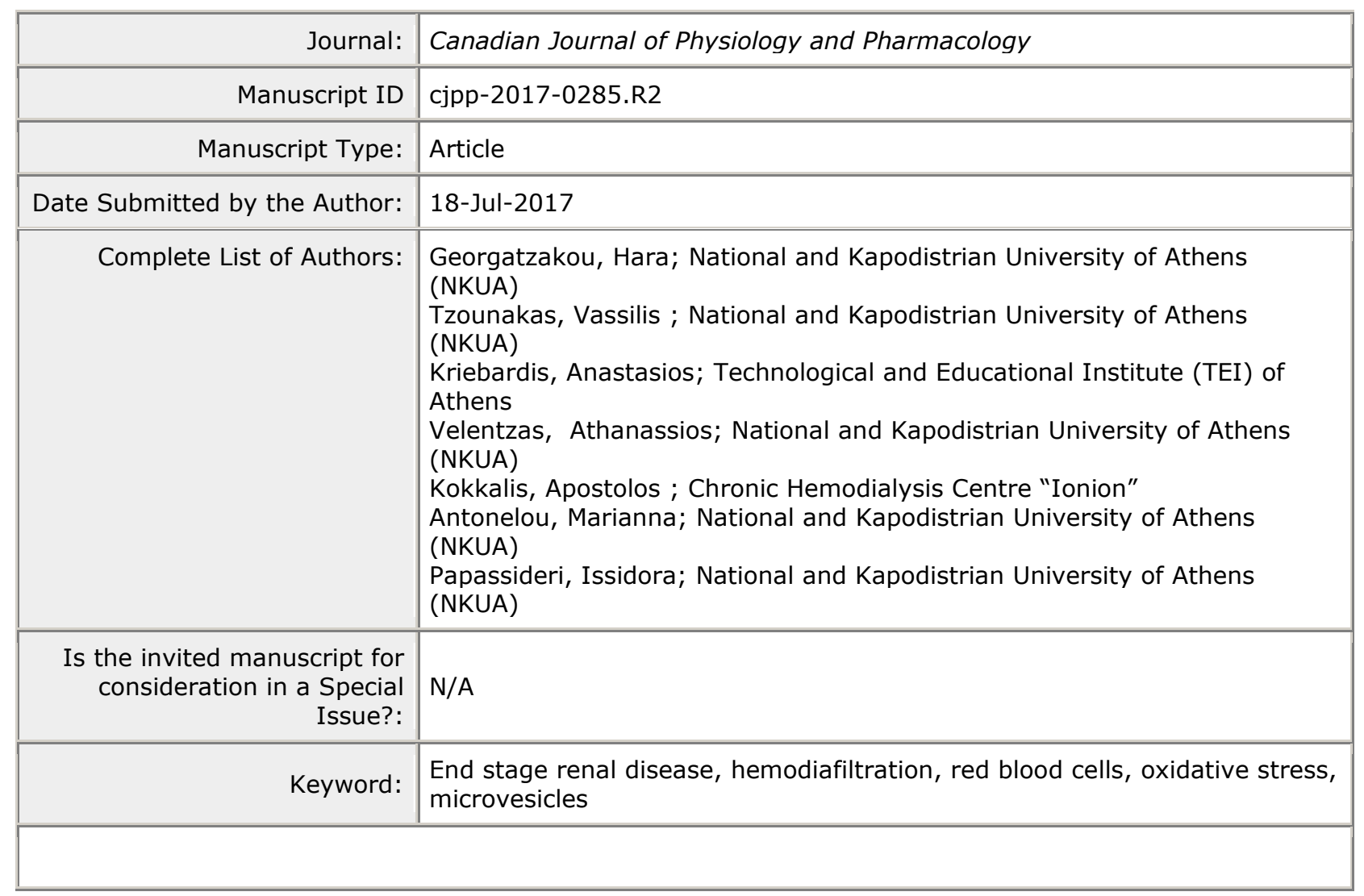


ORIGINAL ARTICLE

Short-term effects of hemodiafiltration versus conventional hemodialysis on erythrocyte performance

Hara T. Georgatzakou, Vassilis L. Tzounakas, Anastasios G. Kriebardis, Athanassios D. Velentzas, Apostolos C. Kokkalis, Marianna H. Antonelou, and Issidora S. Papassideri

H.T. Georgatzakou, Department of Biology, Section of Cell Biology \& Biophysics, School of Science, National and Kapodistrian University of Athens (NKUA), Greece. (email address: xgeorgatzakou@gmail.com)

V.L. Tzounakas, Department of Biology, Section of Cell Biology \& Biophysics, School of Science, National and Kapodistrian University of Athens (NKUA), Greece. (email address: billythecrown@yahoo.com)

A.G. Kriebardis. Department of Medical Laboratories, Faculty of Health and Caring Professions, Technological and Educational Institute (TEI) of Athens, Greece. (email address: akrieb@biol.uoa.gr) A.D. Velentzas, Department of Biology, Section of Cell Biology \& Biophysics, School of Science, National and Kapodistrian University of Athens (NKUA), Greece. (email address: tveletz@biol.uoa.gr)

A.C. Kokkalis. Chronic Hemodialysis Centre "Ionion", Piraeus, Greece. (email address: ackokkalis@hotmail.com)

M.H. Antonelou, Department of Biology, Section of Cell Biology \& Biophysics, School of Science, National and Kapodistrian University of Athens (NKUA), Greece. Corresponding author: Marianna H. Antonelou (email address: manton@biol.uoa.gr).

I.S. Papassideri. Department of Biology, Section of Cell Biology \& Biophysics, School of Science, National and Kapodistrian University of Athens (NKUA), Greece. (email address: ipapasid@biol.uoa.gr) 
Abstract: Hemodiafiltration (HDF) is a renal replacement therapy which is based on the principles of diffusion and convection for the elimination of uremic toxins. A significant and increasing number of end stage renal disease (ESRD) patients are treated with HDF, even in the absence of definite and conclusive survival and anemia treatment data. However, its effects on red blood cell (RBC) physiological features have not been examined in depth. In this study, ESRD patients under regular HDF or conventional hemodialysis (CHD) treatment were examined for RBC-related parameters, including anemia, hemolysis, cell shape, redox status, removal signaling, membrane protein composition and microvesiculation, in repeated paired measurements accomplished before and right after each dialysis session. HDF-group was characterized by better redox potential and suppressed exovesiculation of blood cells compared to the cHD-group pre-dialysis. However, HDF was associated with a temporary but acute, oxidative stress-driven increase in hemolysis, RBC removal signaling and stomatocytosis, probably associated with the effective clearance of dialyzable natural antioxidant components, including uric acid, from the uremic plasma. The nature of these adverse short-term effects of HDF on post-dialysis plasma and RBCs strongly suggests the use of a parallel antioxidant therapy during the HDF session.

Key words: End stage renal disease, hemodiafiltration, hemodialysis, anemia, red blood cells, oxidative stress, stomatocytes, microvesicles, uric acid. 


\section{Introduction}

Accumulation of uremic toxins in the plasma contributes to chronic inflammation, endothelial dysfunction, amyloidosis, oxidative stress and anemia in patients with end stage renal disease (ESRD) (Georgatzakou et al. 2016). Hemodialysis (HD) is a renal replacement therapy that targets to the elimination of uremic toxins and water excess from blood. Conventional hemodialysis (cHD), which provides diffusive clearance of low MW solutes, is the main modality of renal replacement therapy for ESRD patients worldwide. The more recently introduced hemodiafiltration (HDF), which uses a combination of convective and diffusive processes for solute removal, seems to have higher biocompatibility and efficiency in the clearance of uremic solutes across a wider MW range, including middle sized $\beta_{2}$-microglobulin, homocysteine, polyamines and other molecules that influence the endothelial function (Penne et al. 2010). In addition, several studies have suggested that HDF may better control hemodynamic stability, inflammation and iron availability, redox status, erythropoiesis and ESA resistance, and even mortality (probably dose-dependent on the achievement of a critical convection volume), when compared with conventional HD (Maduell et al. 2013; Marcelli et al. 2016; Mercadal et al. 2016). On the other hand, the superiority of this renal replacement therapy compared to the cHD is still a matter of debate, because data on the effectiveness of HDF on several clinical parameters, ranging from anemia to hard endpoints, are conflicting (Karamperis et al. 2005; Susantitaphong et al. 2013; Vilar et al. 2009). In fact, despite the extended (spanning more than two decades) clinical experience with both $\mathrm{CHD}$ and HDF, there are no conclusive evidence from large-scale prospective randomized controlled trials to validate the translatability of the current findings on clinical outcomes, including renal anemia (Ronco 2011; Wiecek and Piecha 2015). In spite of this, there are still no studies reported, describing adverse outcomes to support that HDF is inferior to $\mathrm{CHD}$ in any relevant clinical parameters (Basile et al. 2017). 
Efficient purification of the blood by all relevant uremic toxins, including fluid and salt overload, remains the fundamental objective of all dialysis therapies. However, hematological and metabolic disorders (e.g. anemia, mineral bone disease, oxidative stress etc) that accompany renal failure need to be corrected as well as part of dialysis therapy itself. While red blood cell (RBC) performance as a function of cHD-associated parameters has been studied in ESRD in the past, showing exacerbation of oxidative, metabolic and mechanical stress (Antonelou et al. 2014; Antonelou et al. 2011), the probable effects of HDF on RBC physiology have not been widely assessed. However, this is clinically relevant when considering that: 1) The decreased RBC survival in the hostile, uremic environment (Vos et al. 2011) may be benefited by the clearance adequacy (Ayesh Haj Yousef et al. 2014) or further undermined by several dialysis-related stressful stimuli, through premature aging, eryptosis and other molecular pathways (Abed et al. 2014; Georgatzakou et al. 2016); 2) The results of large clinical trials pointed out that treatment of severe anemia in ESRD by ESAs seems to be more complex that initially appreciated (Wiecek and Piecha 2015); and finally, 3) A significant and increasing number of ESRD patients are treated with HDF, even in the absence of definite and conclusive anemia treatment and survival data. Thus, the present study focused on differences observed in the RBC physiology and performance between HDF- and cHDtreated ESRD patients which are probably related to the management of anemia secondary to ESRD.

\section{Materials and methods}

\section{Subjects}

Thirty two ESRD patients responsive to standard doses of recombinant human erythropoietin supplementation $(9,225 \pm 4,781 \mathrm{IU} /$ week, administrated intravenously at the end of the dialysis session) and twelve, age- and gender-matched, healthy subjects were studied in 
repeated paired tests $(n=3)$ accomplished before and right after dialysis. Sixteen patients had been under high-flux HDF and sixteen patients under low-flux cHD treatment at least for the last three months before examination (Table 1). Blood flow rate was $300 \mathrm{ml} / \mathrm{min}$ vs. $350 \mathrm{ml} / \mathrm{min}$ and dialysate flow rate was $500 \mathrm{ml} / \mathrm{min}$ vs. $700 \mathrm{ml} / \mathrm{min}$ during the $\mathrm{cHD}$ and HDF therapies, respectively. The transmembrane pressures in the dialyzers were $0-50 \mathrm{mmHg}$ vs. $160-230 \mathrm{mmHg}$ for the $\mathrm{cHD}$ and the HDF, respectively. The amount of ultrafiltration varied between patients from $2.5 \mathrm{~L}$ to $3.5 \mathrm{~L}$ per session, but there were not significant differences in the amount of ultrafiltration between the HD and HDF group of patients under investigation. Moreover, there was no change in the dialysis machines (Gambro AK200 or Hospal Integra) and in the dialyzers or membranes used (highly biocompatible, synthetic membranes of polyamix or polyacrilonytrile provided by Gambro) during the time of study. ESRD patients were receiving food supplements (carnitine $6 \mathrm{~g} / \mathrm{wk}, \mathrm{B} 1100 \mathrm{mg} / \mathrm{wk}$, B6 100mg/wk and B12 $1 \mathrm{~g} / \mathrm{wk}$ ), as well as $100 \mathrm{mg} /$ week ferric hydroxide sucrose complex and heparin $(3,050 \pm 1,690 \mathrm{IU} /$ session) intravenously. The primary cause of renal failure was hypertensive nephropathy $(n=2)$, IgA nephropathy $(n=4)$, glomerulonephritis $(n=4)$, polycystic kidney disease $(n=2)$, nephrosclerosis $(n=2)$, nephrolithiasis $(n=2)$ and chronic renal failure of unknown etiology $(n=16)$. Patients were all clinically stable at the time $(\sim 3$ months $)$ of investigation, without clinically appreciated difference in the severity of cardiovascular disease between the HD and HDF groups. In addition, patients with diabetes mellitus, uncontrolled hypertension, active infections, and malignant, inflammatory, autoimmune and hematological diseases, or under blood transfusion were excluded. The study has been submitted and approved by the Research Bioethics and BioSecure Committee of the Department of Biology/NKUA. Investigations were carried out in accordance with the principles of the Declaration of Helsinki. The experiments has been reviewed and approved by the institution's ethics review committee and informed consent was obtained from all blood donors prior to participating in this study. 


\section{Laboratory testing and immunoblotting of RBC membrane proteins}

Hematological analysis, standard biochemical tests in the serum and electrolyte estimation were performed by using automatic blood cell counter and analyzers, respectively. Plasma free hemoglobin $(\mathrm{fHb})$ levels, as an index of intravascular hemolysis, were calculated by the method of Harboe (Harboe 1959). After a double centrifugation of plasma at 1,000 g for $10 \mathrm{~min}$, supernatants were incubated for $30 \mathrm{~min}$ at $20{ }^{\circ} \mathrm{C}$ and absorbance was measured at 380,415 and $450 \mathrm{~nm}$. The formula: 2xOD415 - OD380 - OD450 was used for the calculation of final OD.

Purified leukodepleted RBC fractions were prepared by the method of Beutler (Beutler et al. 1976) and membranes were isolated by RBC lysis with hypotonic (5 mM) sodium phosphate buffer $(\mathrm{pH}$ 8.0) containing protease inhibitors. Equal amounts of membrane protein (15 $\mu \mathrm{g})$ were resolved in $10 \%$ SDS-PAGE gels, electrophoretically transferred onto nitrocellulose membranes and immunoblotted against major membrane proteins (Antonelou et al. 2014). Subsequently, membrane was incubated with the appropriate horseradish peroxidase-conjugated secondary antibody and the immunoreactivity was visualized by enhanced chemiluminescence. The protein bands were quantified in units of intensity by using lengthwise scanning densitometry and an image-processing program (Gel Analyzer v.1.0, Biosure, Athens, Greece).

\section{Redox status of plasma and RBCs}

Total (TAC), uric acid-dependent (UAdAC) and uric acid-independent (UAiAC) antioxidant capacity of plasma were measured by the ferric reducing antioxidant power assay (Benzie and Strain 1996), with/without uricase treatment $\left(0.125 \mathrm{U} / \mathrm{mL}\right.$, for $20 \mathrm{~min}$ at $20^{\circ} \mathrm{C}$ ) (Duplancic et al. 2011). Briefly, plasma was incubated with freshly prepared working FRAP solution (containing $300 \mathrm{mM}$ acetate buffer pH3.6, $10 \mathrm{mM} \mathrm{2,4,6-tripyridyl-s-triazine} \mathrm{in} 40 \mathrm{mM} \mathrm{HCl}$ and $20 \mathrm{mM} \mathrm{FeCl} 3$ in 10:1:1 ratio) and incubated for $4 \mathrm{~min}$ at $37^{\circ} \mathrm{C}$ in a water bath. Absorbance was measured at $593 \mathrm{~nm}$. 
Intracellular accumulation of reactive oxygen species (ROS) with/without stimulation by $100 \mu \mathrm{M}$ tert-butyl hydrperoxide (tBHP) was detected fluorometrically (VersaFluor Fluorometer) by using the fluorescent probe $\mathrm{CMH}_{2}$ DCFDA (Invitrogen), as previously described (Antonelou et al. 2014).

\section{Scanning electron microscopy and flow cytometry analysis of RBCs and microvesicles}

Isolated RBCs were fixed by $2 \%$ glutaraldehyde, post-fixed with $1 \%$ osmium tetroxide, dehydrated in ascending ethanol series and examined in a Philips SEM515 microscope after coating with gold-palladium (Tousimis Samsputter-2a, Rockville, Maryland). RBC shape classification was performed by using standard criteria, as previously adopted (Antonelou et al. 2011).

Enumeration, phenotyping, and phosphatidylserine (PS) exposure on RBCs and microvesicles (MVs) were performed by multicolor flow cytometry using the phycoerythrin (PE)Annexin V apoptosis kit and FITC-conjugated anti-CD235 (BD Pharmingen), as previously described (Tzounakas et al. 2016). MVs were identified by size $(<1 \mu \mathrm{m})$, exposure of RBC-specific markers (anti-CD235) and PS exposure (through annexin $\mathrm{V}$ binding, $\mathrm{AnnV}^{+}$) in plasma isolated after a double $2,500 \mathrm{~g}$ spin of citrated blood at $20^{\circ} \mathrm{C}$.

\section{Statistical analysis}

All experiments have been performed in triplicate. For statistical analysis, the Statistical Package for Social Sciences (IBM SPSS; version 22.0 for Windows IBM Corp., Armonk, NY; administrated by NKUA) was used. Inter-group differences were evaluated by t-test or one-way ANOVA and Mann-Whitney analysis, as appropriate. A Bonferroni correction was used where needed. Pearson's and Spearman's tests were performed to assess correlation $(r)$ between 
parameters following or not normal distribution profiles, respectively. Significance was accepted at $P<0.05$.

\section{Results}

Overall, the ESRD patients were characterized by low RBC count and increased RBC distribution width (RDW). cHD patients exhibited lower hemoglobin (Hb 10.9 $\pm 0.7 \mathrm{~g} / \mathrm{dL}$ ), hematocrit (Hct 33.9 $\pm 1.9 \%$ ), mean corpuscular hemoglobin concentration (MCHC $31.2 \pm 0.6 \mathrm{~g} / \mathrm{dL}$ ) and total ironbinding capacity (TIBC $242 \pm 41 \mu \mathrm{g} / \mathrm{dL}$ ) values compared to patients under HDF, pre-dialysis. At the end of each HDF session, a significant amelioration of the RBC count and indexes (RBC count, Hb, MCV, MCHC and RDW) was observed, whereas in $\mathrm{CHD}$ group only $\mathrm{Hb}$ and $\mathrm{MCHC}$ improved compared to the pre-dialysis levels (Table 1$)$.

In respect to uremia, HDF patients exhibited significantly higher creatinine and uric acid (creatinine: $8.6 \pm 1.2 \mathrm{mg} / \mathrm{dL}$ vs. $6.7 \pm 2.0 \mathrm{mg} / \mathrm{dL} P=0.023$; uric acid: $7.9 \pm 1.8 \mathrm{mg} / \mathrm{dL}$ vs. $6.1 \pm 1.0 \mathrm{mg} / \mathrm{dL}$ $P=0.009$, respectively) along with a trend for higher urea levels ( $143 \pm 23 \mathrm{vs.} 123 \pm 27 \mathrm{mg} / \mathrm{dL} P=0.110$, respectively), compared to cHD patients, in spite of the better urea reduction ratio ( $78 \pm 6 \%$ vs. $69 \pm 7 \% P=0.007$ for HDF vs. cHD, respectively) (Table 1). High pre-dialysis levels of uric acid in HDF patients were reflected in the elevated urate-dependent antioxidant capacity (UAdAC) of plasma, which decreased significantly post-dialysis in both groups (Table 1).

ESRD RBCs were more susceptible to phosphatidylserine (PS) externalization than healthy RBCs, independently of the dialysis technique applied. Yet, the percentage $\mathrm{PS}^{+} \mathrm{RBC}$ was significantly lower $(P=0.026)$ in HDF than in $\mathrm{CHD}$ patients before dialysis $(1.13 \pm 0.38 \%$ vs $1.73 \pm 0.67 \%$, respectively) (Figure $1 \mathrm{~A}$ ). Of note, dialysis method had a different impact on PS exposure on RBCs, since HDF increased it significantly (by $40 \%, P=0.031$ ) compared to the predialysis levels, in opposite to the cHD (Figure 1A). Likewise, HDF exhibited a negative effect on 
baseline hemolysis (increase from $15.1 \pm 6.6 \mathrm{mg} / \mathrm{dL}$ to $17.9 \pm 7.4 \mathrm{mg} / \mathrm{dL}, P=0.026$ vs controls, Figure 1B), intracellular ROS accumulation (from $457 \pm 201$ to $480 \pm 162 \mathrm{RFU}, P=0.011$ vs controls, Figure 1C), and on exogenously-induced intracellular ROS levels (from $630 \pm 273$ to $717 \pm 232 \mathrm{RFU}, P=0.009$ vs controls, Figure 1D), in striking contrast to a trend for amelioration (fHb, Figure $1 \mathrm{~B}$ ) or neutral effect (ROS, Figure 1C and 1D) of cHD on those physiological features.

Examination of RBC shape, revealed a trend for stomatocytic transformation in HDF RBCs pre-dialysis that was actively promoted by the dialysis session, resulting in significantly higher percentage of stomatocytes in HDF patients compared to the $\mathrm{cHD}$ patients post-dialysis $(8.8 \pm 2.6 \%$ vs $6.2 \pm 2.1 \%$, respectively, $P=0.023$ ) (Figure $2 \mathrm{~A}$ and $\mathrm{B}$ ). Stomatocytosis was positively correlated with serum uric acid levels $(r=0.810, P=0.004)$ but negatively with the percentage of PS ${ }^{+}$BBCs $(r=-$ $0.684, P=0.043)$ in HDF patients pre-dialysis.

As expected, the uremic plasma was further characterized by pathological accumulation of total (MVs) and RBC-derived (R-MVs) circulating microvesicles (Figure 2C, left panel). Both populations of extracellular vesicles were significantly lesser in the HDF (MVs: 9,330 $\pm 4,813$

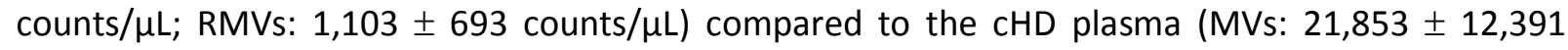
counts/ $\mu \mathrm{L} ; \mathrm{RMVs}: 2,365 \pm 1,691$ counts $/ \mu \mathrm{L})$ pre-dialysis $(P=0.024$ and $P=0.043$, respectively). Postdialysis, the overall MVs population decreased to normal size (HDF: 4,077 $\pm 2,275$ counts/ $\mu \mathrm{L}$; cHD: $5,032 \pm 3,439$ counts $/ \mu \mathrm{L}$ ), while the RBC-derived vesicles remained at high levels (HDF: 1,214 \pm 952 ; cHD: $1,044 \pm 627$ vs. healthy subjects $130 \pm 85$ counts/ $\mu \mathrm{L}$ ) (Figure $2 \mathrm{C}$, right panel). In many patients treated by $\mathrm{CHD}$, a significant reduction in R-MVs compared to the pre-dialysis levels was recorded, however, that reduction was not a statistically significant feature of cHD-group $(P=0.080)$ owing to high patient-to-patient variation.

Finally, the RBC membrane of HDF-treated patients was characterized by severe deficiency in aquaporin $1(47 \pm 7 \%, P=0.025)$ and overexpression of $\mathrm{Hsp} 70(254 \pm 31 \%, P=0.015)$ compared to 
healthy subjects $(100 \%)$, but also, by significantly lower levels of stomatin compared to the membrane of cHD patients pre-dialysis ( $81 \pm 11 \%$ vs. $126 \pm 10 \%$, respectively, $P=0.009$ ). The RBC membrane of cHD-treated subjects on the other side was severely deficient in CD47 (46 $\pm 11 \%$, $P=0.004)$ but over-expressed Glut $1(251 \pm 38 \%, P=0.016)$ and IgGs $(693 \pm 211 \%, P=0.033)$ molecules (Figure 3A). Following an HDF session, increased membrane binding of oxidized/denatured Hb was observed (182 $\pm 68 \%, P=0.047$ ) (Figure 3B bar-graph and insert). cHD seemed to be associated with an overall reduction in the expression level of several membrane proteins, including stomatin (77 $\pm 9 \%, P=0.005$, Figure $3 \mathrm{~B})$. In some cases, that reduction tended to regularity (as in the case of Glut 1 ) but in other, cHD leaded to protein deficiency (aquaporin $1,62 \pm 8 \%, P=0.044$ ) or just exacerbated an existing shortage (CD47, from $46 \pm 11 \%$ to $23 \pm 5 \%, P=0.010$ ).

\section{Discussion}

Reduced lifespan and pathologic modifications in circulating RBCs arising from various types of uremic and dialysis-induced stresses, contribute to anemia and other hematological complications in ESRD. While conventional hemodialysis has been studied in respect to its probable effects on RBC morphology and physiology (Antonelou et al. 2014; Antonelou et al. 2011), whether and to what extent hemodiafiltration represents a stress factor for blood cells, or whether it is more "friendly" for them as a renal replacement therapy compared to the cHD, is largely unknown. The present comparative study assessed an array of functionally important physiological features of circulating RBCs in patients regularly treated by either HDF or cHD.

According to the analysis, greater urea reduction ratio was recorded in patients treated by HDF compared to cHD, in spite of their worse basal toxin levels. Hemodialysis adequacy is expected to have a beneficial effect on anemia. As a probable result of the better clearance of uremic compounds, which are supposed to suppress bone marrow's response to erythropoietin (Locatelli 
and Del Vecchio 2003) and to trigger inflammatory responses (Rossi et al. 2014), HDF patients exhibited on average better RBC indexes and lower levels of inflammation markers, such as TIBC and RDW, compared to the CHD patients before dialysis. Variability in RBC volume (RDW index) and low TIBC have been widely associated with protein-energy wasting, inflammation, poor quality of life, cardiovascular events and mortality in hemodialysis patients, in both prospective and retrospective observational studies (Bross et al. 2009; Lippi et al. 2009; Vashistha et al. 2016). On a largely similar clinical ground of renal disease, time on dialysis, Epo responsiveness and dose, vitamin supplementation and co-morbidities in cHD and HDF patient groups, our results are rather consistent with studies reporting beneficial effects of chronic HDF over cHD in ESRD, continuing, thus, the controversy in the field (Bowry and Gatti 2011; Locatelli et al. 2012; Schneider et al. 2012; Susantitaphong et al. 2013) .

At RBC level, low levels of intravascular hemolysis, irreversible RBC shape modifications and intracellular oxidative stress were detected in patients treated by either cHD or HDF. However, the HDF-treated patients exhibited lower percentage of $\mathrm{PS}^{+}$circulating RBCs and microvesicles and almost normal protein composition of the RBC membrane compared with the cHD patients predialysis. PS exposure on RBC surface, a common finding in ESRD RBCs (Abed et al. 2014; Bonomini et al. 1999), is a sign of eryptosis (namely, of premature suicidal RBC death) and a potent recognition signal leading to accelerated erythrophagocytosis, and, thus, susceptibility to anemia. Moreover, accumulation of microvesicles has been implicated in inflammation and coagulation (including intraglomerular coagulation) issues in uremic patients (Ando et al. 2002; He et al. 2016), and consequently, a lower vesiculation rate might be associated with the previously reported better endothelial function (Jia et al. 2016) and reduced cardiovascular mortality rates in HDF- vs. cHD-treated patients (Daugirdas 2016; Maduell et al. 2013). Finally, at RBC membrane level, the RBCs of the HDF-treated patients were only characterized by over-expression of Hsp70 (a molecular 
chaperone protective for membrane peripheral proteins (Biondani et al. 2008)) and deficiency in aquaporin 1 (the membrane water channel responsible for changes in RBC volume in response to the tonicity of the medium). Aquaporin-1 deficiency might be associated with the effects of erythropoietin on cell water regulation (Rentsch et al. 2006) and/or, with the stomatocytic transformation of RBCs, as discussed below. On the other hand, the RBC membrane of the cHDtreated patients was characterized by several disturbances, including over-accumulation of plasma IgGs, overexpression of glucose transporter 1 and sharp deficiency in the "marker-of-self" CD47. These modifications are indicative of: a) metabolic and probably oxidative stress, as glucose transporter 1 mediates the transport of both glucose and dehydroascorbic acid molecules into RBCs (Montel-Hagen et al. 2008), as well as b) susceptibility of cHD-RBCs to opsonization (IgGs binding) and erythrophagocytosis, since a severe deficiency of the membrane in CD47 may induce RBC clearance through the SIRPalpha protein receptor in macrophages (Lutz and Bogdanova 2013).

On the other side, the effective elimination of uremic compounds by the HDF brings stressful, though temporary, challenges to the RBCs of ESRD patients, which undermine their functional capacity soon after the dialysis session. Indeed, post-HDF, the uric acid-dependent antioxidant capacity of plasma fell to very low levels compared to the plasma post-cHD, as a result of the better clearance of the dialyzable uric acid. Nevertheless, apart from being a uremic toxin, the end product of purine metabolism in human is a potent natural antioxidant factor, that protects both plasma (in the antioxidant capacity of which it contributes by $35-65 \%$ ) and cells from oxidative damages, through its free radical scavenging capacity (Assis et al. 2015; Tzounakas et al. 2015). Of note, either as an indicator of better nutritional status or not, low UA has been proposed as a mortality risk factor in HD patients (Beberashvili et al. 2015) and more recently, it was found that $\mathrm{RBC}$ life span (that is a significant measure of/contributor to anemia) in dialysis patients is positively correlated with levels of uric acid and blood urea nitrogen (Ma et al. 2017). The high clearance 
potential of HDF probably results in the acute elimination of additional dilute factors that also function in strengthening the antioxidant defense reactions in plasma, including albumin (Ahrenholz et al. 2004), vitamins (Morena et al. 2002) and trace elements (Prodanchuk et al. 2014). Trace elements are cofactors for many antioxidant enzymes of blood (Chan et al. 1998), albumin is a main antioxidant protein of plasma contributing to its free-radical trapping capacity, whereas hydrophobic vitamin C helps vitamin $\mathrm{E}$ to work in lipid detoxification.

Thus, while in general, dialysis therapy per se seems to negatively affect the systemic redox status and the RBC antioxidant defenses (Poulianiti et al. 2016), HDF in particular, seemed to be associated with an additional temporary acute oxidative stress. The effective elimination of "waste" natural antioxidant factors from the uremic plasma by the HDF is manifested in the pathological levels of ROS accumulation and susceptibility to oxidative stimuli that were exclusively observed in HDF-RBCs right after the completion of the dialysis session. Oxidative stress of that origin, was further highlighted by the accumulation of oxidized/denatured $\mathrm{Hb}$ to the RBC membrane, the acute increase in PS externalization, the increased intravascular hemolysis (Quaye 2015) and, probably, by the appearance of transient stomatocytosis in HDF-treated patients. Eryptosis, which is strongly associated with oxidative stress and ceramide formation in uremia, is further triggered by the dialysis procedure (Abed et al. 2014), while deficiency in reduced GSH has been related to higher risk of hemolysis in ESRD (Weinstein et al. 2000). The tripeptide GSH is an important antioxidant molecule that can easily pass through filters' pores, especially in HDF, where convection reinforces the removal of substances. The release of free, extracellular $\mathrm{Hb}$ and its degradation products in post-dialysis plasma is expected to induce oxidative stress in HDF-blood, as a part of a toxic feedback loop (Quaye 2015). Post-HDF RBC hemolysis and PS exposure, a marker of oxidative stress for RBCs (Mandal et al. 2002), might also represent adverse effects of that temporary increase in oxidative stimuli. Appearance of stomatocytes, which represent reversible 
RBC modifications, has been also related with various oxidative and inflammatory states (Gyawali et al. 2015). Stomatocytes are generally considered as the morphological change adopted by erythrocytes during their response to oxidants (Vota et al. 2013). Notably, in our patients, the stomatocytic transformation of RBCs was positively correlated with serum uric acid levels and seemed to be a feature of the HDF-group by many ways: HDF-RBCs were characterized by lower membrane expression of stomatin and its membrane protein partners (Rungaldier et al. 2013) aquaporin 1 and glucose transporter 1, compared to the cHD-RBCs, and, moreover, stomatocytosis was triggered by HDF and not by $\mathrm{cHD}$. HDF-associated membrane-perturbing cationic factors might also induce a stomatocytic transformation of RBC morphology through preferential accumulation in, and therefore expansion of, the inner (relative to the outer) leaflet of the lipid bilayer (Reinhart and Chien 1987). Stomatocytosis is expected to affect cation permeability and intrinsic deformability of the membrane, as well as RBC aggregation and filterability in the microcirculation of HDF-treated ESRD group post-dialysis (Chabanel et al. 1987; Reinhart and Chien 1986).

Moreover, uremia and dialysis-associated stresses trigger exovesiculation in blood cells (Boulanger et al. 2007). Our patients (especially those of the cHD group) were characterized by over-accumulation of MVs to plasma, compared to healthy subjects. Dialysis lowered the mixed population of plasma MVs (where MVs of platelet, RBC, leukocyte, and endothelial origin are found) to normal levels, but it had minor effects on RBC-derived MVs, especially in the HDF group, suggesting dialysis-triggered neo-vesiculation of RBC membrane. To support this assumption, a trend for lower post-dialysis R-MVs levels was observed in cHD-treated patients compared to the pre-dialysis levels (despite at no statistically significant point due to high inter-patient variability), in whom post-dialysis oxidative stress (an established trigger of vesiculation in RBCs) remained at normal levels, in opposite to the HDF-treated patients. Moreover, the increased stomatocytic 
transformation seen in HDF-RBCs post-dialysis is expected to inhibit exovesiculation of the membrane (Schreier et al. 2000).

Although the time-course evaluation of this effect needs further studies of HDF-treated patients post-dialysis, our results suggest that application of HDF should be combined with antioxidant protection of blood during the dialysis or soon after it, to mitigate the increases in intravascular hemolysis, RBC removal signaling and exovesiculation. Oral or intravenous supplementation of reduced glutathione, L-carnitine and vitamin C has been shown to improve lifespan, metabolism and other physiological properties of RBCs (Candan and Gultekin 2002; Usberti et al. 1997). In addition, intravenous infusion of reduced GSH or vitamin C soon after dialysis by vitamin-E coated membranes was shown to improve anemia and ameliorate erythropoietin requirements in hemodialysis patients (D'Arrigo et al. 2017; Usberti et al. 2002; Yang et al. 2006).

To our knowledge, this study is the first to evaluate the effects of HDF on overall RBC biology and performance in ESRD patients. However, there are several limitations. First, the patient population was relatively small and thus some measurements that obviously differed between groups but exhibited high inter-patient variation were not found statistically significant. To overcome that limitation, we tried to construct as much as possible homogeneous patient groups (in terms of demographic and clinical data) and moreover, we performed repeated paired measurements (at least three per patient) before and soon after the dialysis session to increase the statistical power of the study. Second, direct inflammation markers such as high sensitivity Creactive protein and interleukins were not measured and thus our results cannot be directly compared to those of other studies reporting the inflammation state of cHD- or HDF-treated ESRD patients on the basis of those classic measurements. Instead of it, alternative but also widely recognized markers of inflammation (RDW, TIBC) were used. Third, despite the fact that the two 
groups of patients did not differ significantly at baseline, age or time on dialysis treatment were not completely balanced between them, as a result of efforts to construct as possible as larger groups of clinically "equal" (in respect to comorbidities, ESA dose and responsiveness etc) patients. Thus, a possible interference of those variables with our results on differences between CHD and HDF cannot be excluded. Fourth, the pre - post treatment values of free $\mathrm{Hb}$, microvesicles and other variables were not adjusted for the amount of ultrafiltration since we had only data for the average amount of ultrafiltration per patient and not for each treatment individually. Despite that, the amount of ultrafiltration exhibited very little intra-patient variation between sessions and no statistical difference between the groups. Finally, evaluation of the intracellular antioxidant activity in HDF vs. CHD RBCs and time-course recording of the RBC performance in HDF patients postdialysis were not performed and thus, the time when the redox status is balanced in those patients cannot be estimated. The potential short- and long-term adverse or beneficial effects of HDF on the RBCs must be tested by controlled clinical trials before recommendations can be made for clinical practice.

\section{Conclusions}

Taken together, similar low levels of baseline intravascular hemolysis, irreversible RBC shape modifications and intracellular oxidative stress were detected in clinically stable ESRD patients treated by cHD or HDF. RBC removal signaling (PS, CD47, IgGs etc) and membrane vesiculation, which might underlie susceptibility to anemia, inflammation, thrombosis and endothelial damages in ESRD, were lower in the RBCs of HDF-treated patients compared to cHD counterparts. The beneficial effects of HDF on RBCs are diluted by a temporary acute oxidative stress that undermines their functional capacity soon after the dialysis session, as a probable result of "over-eliminating" dialyzable natural antioxidant factors, including uric acid, from the uremic plasma. 


\section{Conflict of Interest}

None declared.

\section{Acknowledgements}

We thank all patients and healthy blood volunteers; we are extremely grateful to Mrs. Vasileia Stoupa, Head nurse in the Chronic Hemodialysis Centre "Ionion" for kindly organizing blood donations in a way exhibiting high professional standards, responsibility and consistency along with excellent collaboration skills.

\section{References}

Abed, M., Artunc, F., Alzoubi, K., Honisch, S., Baumann, D., Foller, M. et al. 2014. Suicidal erythrocyte death in end-stage renal disease. J Mol Med (Berl) 92(8): 871-879. doi: 10.1007/s00109-014-1151-4.

Ahrenholz, P.G., Winkler, R.E., Michelsen, A., Lang, D.A., and Bowry, S.K. 2004. Dialysis membranedependent removal of middle molecules during hemodiafiltration: the beta2microglobulin/albumin relationship. Clin Nephrol 62(1): 21-28.

Ando, M., Iwata, A., Ozeki, Y., Tsuchiya, K., Akiba, T., and Nihei, H. 2002. Circulating plateletderived microparticles with procoagulant activity may be a potential cause of thrombosis in uremic patients. Kidney Int 62(5): 1757-1763. doi: kid627 [pii]10.1046/j.1523-1755.2002.00627.x. Antonelou, M.H., Georgatzakou, H.T., Tzounakas, V.L., Velentzas, A.D., Kokkalis, A.C., Kriebardis et al. 2014. Blood modifications associated with end stage renal disease duration, progression and cardiovascular mortality: a 3-year follow-up pilot study. J Proteomics 101: 88-101. doi: 10.1016/j.jprot.2014.02.009S1874-3919(14)00058-X [pii]. 
Antonelou, M.H., Kriebardis, A.G., Velentzas, A.D., Kokkalis, A.C., Georgakopoulou, S.C., and Papassideri, I.S. 2011. Oxidative stress-associated shape transformation and membrane proteome remodeling in erythrocytes of end stage renal disease patients on hemodialysis. J Proteomics 74(11): 2441-2452. doi: 10.1016/j.jprot.2011.04.009S1874-3919(11)00151-5 [pii].

Assis, R.P., Castro, J.F., Gutierres, V.O., Arcaro, C.A., Brotto, R.S., Oliveira et al. 2015. Effects of uremic solutes on reactive oxygen species in vitro model systems as a possibility of support the renal function management. BMC Nephrol 16: 50 . doi: 10.1186/s12882-015-0029110.1186/s12882-015-0029-1 [pii].

Ayesh Haj Yousef, M.H., Bataineh, A., Elamin, E., Khader, Y., Alawneh, K., and Rababah, M. 2014. Adequate hemodialysis improves anemia by enhancing glucose-6-phosphate dehydrogenase activity in patients with end-stage renal disease. BMC Nephrol 15: 155. doi: 10.1186/1471-236915-1551471-2369-15-155 [pii].

Basile, C., Davenport, A., and Blankestijn, P.J. 2017. Why choose high volume online post-dilution hemodiafiltration? J Nephrol 30(2): 181-186. doi: 10.1007/s40620-016-0343-010.1007/s40620016-0343-0 [pii].

Beberashvili, I., Sinuani, I., Azar, A., Shapiro, G., Feldman, L., Stav, K. et al. 2015. Serum uric acid as a clinically useful nutritional marker and predictor of outcome in maintenance hemodialysis patients. Nutrition 31(1): 138-147. doi: 10.1016/j.nut.2014.06.012S0899-9007(14)00333-5 [pii].

Benzie, I.F., and Strain, J.J. 1996. The ferric reducing ability of plasma (FRAP) as a measure of "antioxidant power": the FRAP assay. Anal Biochem 239(1): 70-76. doi: S0003-2697(96)90292-4 [pii]10.1006/abio.1996.0292.

Beutler, E., West, C., and Blume, K.G. 1976. The removal of leukocytes and platelets from whole blood. J Lab Clin Med 88(2): 328-333. doi: 0022-2143(76)90381-4 [pii].

Biondani, A., Turrini, F., Carta, F., Matte, A., Filippini, A., Siciliano, A., et al. 2008. Heat-shock protein-27, -70 and peroxiredoxin-II show molecular chaperone function in sickle red cells: 
Evidence from transgenic sickle cell mouse model. Proteomics Clin Appl 2(5): 706-719. doi: 10.1002/prca.200780058.

Bonomini, M., Sirolli, V., Settefrati, N., Dottori, S., Di Liberato, L., and Arduini, A. 1999. Increased erythrocyte phosphatidylserine exposure in chronic renal failure. J Am Soc Nephrol 10(9): 19821990.

Boulanger, C.M., Amabile, N., Guerin, A.P., Pannier, B., Leroyer, A.S., Mallat, C.N., et al. 2007. In vivo shear stress determines circulating levels of endothelial microparticles in end-stage renal disease. Hypertension 49(4): 902-908. doi: 01.HYP.0000259667.22309.df [pii]10.1161/01.HYP.0000259667.22309.df.

Bowry, S.K., and Gatti, E. 2011. Impact of hemodialysis therapy on anemia of chronic kidney disease: the potential mechanisms. Blood Purif 32(3): 210-219. doi: 10.1159/000329573000329573 [pii].

Bross, R., Zitterkoph, J., Pithia, J., Benner, D., Rambod, M., Kovesdy, C.P., et al. 2009. Association of serum total iron-binding capacity and its changes over time with nutritional and clinical outcomes in hemodialysis patients. Am J Nephrol 29(6): 571-581. doi: 10.1159/000191470000191470 [pii].

Candan, F., and Gultekin, F. 2002. Effect of vitamin C and zinc on osmotic fragility and lipid peroxidation in zinc-deficient haemodialysis patients. Cell Biochem Funct 20(2): 95-98. doi: 10.1002/cbf.947.

Chabanel, A., Reinhart, W., and Chien, S. 1987. Increased resistance to membrane deformation of shape-transformed human red blood cells. Blood 69(3): 739-743.

Chan, S., Gerson, B., and Subramaniam, S. 1998. The role of copper, molybdenum, selenium, and zinc in nutrition and health. Clin Lab Med 18(4): 673-685.

D'Arrigo, G., Baggetta, R., Tripepi, G., Galli, F., and Bolignano, D. 2017. Effects of Vitamin E-Coated versus Conventional Membranes in Chronic Hemodialysis Patients: A Systematic Review and MetaAnalysis. Blood Purif 43(1-3): 101-122. doi: 10.1159/000453444000453444 [pii]. 
Daugirdas, J.T. 2016. Lower cardiovascular mortality with high-volume hemodiafiltration: a cool effect? Nephrol Dial Transplant 31(6): 853-856. doi: 10.1093/ndt/gfv412gfv412 [pii].

Duplancic, D., Kukoc-Modun, L., Modun, D., and Radic, N. 2011. Simple and rapid method for the determination of uric acid-independent antioxidant capacity. Molecules 16(8): 7058-7068. doi: 10.3390/molecules16087058molecules16087058 [pii].

Georgatzakou, H.T., Antonelou, M.H., Papassideri, I.S., and Kriebardis, A.G. 2016. Red blood cell abnormalities and the pathogenesis of anemia in end-stage renal disease. Proteomics Clin Appl 10(8): 778-790. doi: 10.1002/prca.201500127.

Gyawali, P., Richards, R.S., Bwititi, P.T., and Nwose, E.U. 2015. Association of abnormal erythrocyte morphology with oxidative stress and inflammation in metabolic syndrome. Blood Cells Mol Dis 54(4): 360-363. doi: 10.1016/j.bcmd.2015.01.005S1079-9796(15)00025-X [pii].

Harboe, M. 1959. A method for determination of hemoglobin in plasma by near-ultraviolet spectrophotometry. Scand J Clin Lab Invest 11: 66-70. doi: 10.3109/00365515909060410.

He, Z., Zhang, Y., Cao, M., Ma, R., Meng, H., Yao, Z. et al. 2016. Increased phosphatidylserineexposing microparticles and their originating cells are associated with the coagulation process in patients with IgA nephropathy. Nephrol Dial Transplant 31(5): 747-759. doi: 10.1093/ndt/gfv403gfv403 [pii].

Jia, P., Jin, W., Teng, J., Zhang, H., Zou, J., Liu, Z. et al. 2016. Acute Effects of Hemodiafiltration Versus Conventional Hemodialysis on Endothelial Function and Inflammation: A Randomized Crossover Study. Medicine (Baltimore) 95(16): e3440. doi: 10.1097/MD.000000000000344000005792-201604190-00032 [pii].

Karamperis, N., Sloth, E., and Jensen, J.D. 2005. Predilution hemodiafiltration displays no hemodynamic advantage over low-flux hemodialysis under matched conditions. Kidney Int 67(4): 1601-1608. doi: S0085-2538(15)50620-0 [pii]10.1111/j.1523-1755.2005.00242.x.

Lippi, G., Targher, G., Montagnana, M., Salvagno, G.L., Zoppini, G., and Guidi, G.C. 2009. Relation between red blood cell distribution width and inflammatory biomarkers in a large cohort of 
unselected outpatients. Arch Pathol Lab Med 133(4): 628-632. doi: 10.1043/1543-2165133.4.6282008-0279-OAR1 [pii].

Locatelli, F., Altieri, P., Andrulli, S., Sau, G., Bolasco, P., Pedrini, L.A., et al. 2012. Predictors of haemoglobin levels and resistance to erythropoiesis-stimulating agents in patients treated with low-flux haemodialysis, haemofiltration and haemodiafiltration: results of a multicentre randomized and controlled trial. Nephrol Dial Transplant 27(9): 3594-3600. doi: 10.1093/ndt/gfs117gfs117 [pii].

Locatelli, F., and Del Vecchio, L. 2003. Dialysis adequacy and response to erythropoietic agents: what is the evidence base? Nephrol Dial Transplant 18 Suppl 8: viii29-35.

Lutz, H.U., and Bogdanova, A. 2013. Mechanisms tagging senescent red blood cells for clearance in healthy humans. Front Physiol 4: 387. doi: 10.3389/fphys.2013.00387.

Ma, J., Dou, Y., Zhang, H., Thijssen, S., Williams, S., Kuntsevich, V. et al. 2017. Correlation between Inflammatory Biomarkers and Red Blood Cell Life Span in Chronic Hemodialysis Patients. Blood Purif 43(1-3): 200-205. doi: 10.1159/000452728000452728 [pii].

Maduell, F., Moreso, F., Pons, M., Ramos, R., Mora-Macia, J., Carreras, J. et al. 2013. Highefficiency postdilution online hemodiafiltration reduces all-cause mortality in hemodialysis patients. J Am Soc Nephrol 24(3): 487-497. doi: 10.1681/ASN.2012080875ASN.2012080875 [pii].

Mandal, D., Moitra, P.K., Saha, S., and Basu, J. 2002. Caspase 3 regulates phosphatidylserine externalization and phagocytosis of oxidatively stressed erythrocytes. FEBS Lett 513(2-3): 184-188. doi: S0014579302022949 [pii].

Marcelli, D., Bayh, I., Merello, J.I., Ponce, P., Heaton, A., Kircelli, F. et al. 2016. Dynamics of the erythropoiesis stimulating agent resistance index in incident hemodiafiltration and high-flux hemodialysis patients. Kidney Int 90(1): 192-202. doi: 10.1016/j.kint.2016.03.009S00852538(16)30056-4 [pii].

Mercadal, L., Franck, J.E., Metzger, M., Urena Torres, P., de Cornelissen, F., Edet, S. et al. 2016. Hemodiafiltration Versus Hemodialysis and Survival in Patients With ESRD: The French Renal 
Epidemiology and Information Network (REIN) Registry. Am J Kidney Dis 68(2): 247-255. doi: 10.1053/j.ajkd.2015.11.016S0272-6386(15)01436-5 [pii].

Montel-Hagen, A., Kinet, S., Manel, N., Mongellaz, C., Prohaska, R., Battini et al. 2008. Erythrocyte Glut1 triggers dehydroascorbic acid uptake in mammals unable to synthesize vitamin C. Cell 132(6): 1039-1048. doi: 10.1016/j.cell.2008.01.042S0092-8674(08)00204-3 [pii].

Morena, M., Cristol, J.P., Bosc, J.Y., Tetta, C., Forret, G., Leger, C.L. et al. 2002. Convective and diffusive losses of vitamin C during haemodiafiltration session: a contributive factor to oxidative stress in haemodialysis patients. Nephrol Dial Transplant 17(3): 422-427.

Penne, E.L., van der Weerd, N.C., Blankestijn, P.J., van den Dorpel, M.A., Grooteman, M.P., Nube, M.J. et al. 2010. Role of residual kidney function and convective volume on change in beta2microglobulin levels in hemodiafiltration patients. Clin J Am Soc Nephrol 5(1): 80-86. doi: 10.2215/CJN.03340509CJN.03340509 [pii].

Poulianiti, K.P., Kaltsatou, A., Mitrou, G.I., Jamurtas, A.Z., Koutedakis, Y., Maridaki, M. et al. 2016. Systemic Redox Imbalance in Chronic Kidney Disease: A Systematic Review. Oxid Med Cell Longev 2016: 8598253. doi: 10.1155/2016/8598253.

Prodanchuk, M., Makarov, O., Pisarev, E., Sheiman, B., and Kulyzkiy, M. 2014. Disturbances of trace element metabolism in ESRD patients receiving hemodialysis and hemodiafiltration. Cent European J Urol 66(4): 472-476. doi: 10.5173/ceju.2013.04.art2300307 [pii].

Quaye, I.K. 2015. Extracellular hemoglobin: the case of a friend turned foe. Front Physiol 6: 96. doi: 10.3389/fphys.2015.00096.

Reinhart, W.H., and Chien, S. 1986. Red cell rheology in stomatocyte-echinocyte transformation: roles of cell geometry and cell shape. Blood 67(4): 1110-1118.

Reinhart, W.H., and Chien, S. 1987. Echinocyte-stomatocyte transformation and shape control of human red blood cells: morphological aspects. Am J Hematol 24(1): 1-14. 
Rentsch, R.L., Damsgaard, R., Lundby, C., and Juel, C. 2006. Effects of darbepoetin injections on erythrocyte membrane transport protein expressions in humans. J Appl Physiol (1985) 101(1): 164-168. doi: 01376.2005 [pii]10.1152/japplphysiol.01376.2005.

Ronco, C. 2011. Hemodiafiltration: Evolution of a technique towards better dialysis care. Contrib Nephrol 168: 19-27. doi: 10.1159/000321741000321741 [pii].

Rossi, M., Campbell, K.L., Johnson, D.W., Stanton, T., Vesey, D.A., Coombes, J.S. et al. 2014. Protein-bound uremic toxins, inflammation and oxidative stress: a cross-sectional study in stage 34 chronic kidney disease. Arch Med Res 45(4): 309-317. doi: 10.1016/j.arcmed.2014.04.002S01884409(14)00051-4 [pii].

Rungaldier, S., Oberwagner, W., Salzer, U., Csaszar, E., and Prohaska, R. 2013. Stomatin interacts with GLUT1/SLC2A1, band 3/SLC4A1, and aquaporin-1 in human erythrocyte membrane domains. Biochim Biophys Acta 1828(3): 956-966. doi: 10.1016/j.bbamem.2012.11.030S00052736(12)00421-X [pii].

Schneider, A., Drechsler, C., Krane, V., Krieter, D.H., Scharnagl, H., Schneider, M.P. et al. 2012. The effect of high-flux hemodialysis on hemoglobin concentrations in patients with CKD: results of the MINOXIS study. Clin J Am Soc Nephrol 7(1): 52-59. doi: 10.2215/CJN.02710311CJN.02710311 [pii].

Schreier, S., Malheiros, S.V., and de Paula, E. 2000. Surface active drugs: self-association and interaction with membranes and surfactants. Physicochemical and biological aspects. Biochim Biophys Acta 1508(1-2): 210-234. doi: S0304-4157(00)00012-5 [pii].

Susantitaphong, P., Siribamrungwong, M., and Jaber, B.L. 2013. Convective therapies versus lowflux hemodialysis for chronic kidney failure: a meta-analysis of randomized controlled trials. Nephrol Dial Transplant 28(11): 2859-2874. doi: 10.1093/ndt/gft396gft396 [pii].

Tzounakas, V.L., Georgatzakou, H.T., Kriebardis, A.G., Papageorgiou, E.G., Stamoulis, K.E., Foudoulaki-Paparizos, L.E. et al. 2015. Uric acid variation among regular blood donors is indicative of red blood cell susceptibility to storage lesion markers: A new hypothesis tested. Transfusion 55(11): 2659-2671. doi: 10.1111/trf.13211. 
Tzounakas, V.L., Georgatzakou, H.T., Kriebardis, A.G., Voulgaridou, A.I., Stamoulis, K.E., Foudoulaki-Paparizos, L.E. et al. 2016. Donor variation effect on red blood cell storage lesion: a multivariable, yet consistent, story. Transfusion 56(6): 1274-1286. doi: 10.1111/trf.13582.

Usberti, M., Gerardi, G., Micheli, A., Tira, P., Bufano, G., Gaggia, P. et al. 2002. Effects of a vitamin E-bonded membrane and of glutathione on anemia and erythropoietin requirements in hemodialysis patients. J Nephrol 15(5): 558-564.

Usberti, M., Lima, G., Arisi, M., Bufano, G., D'Avanzo, L., and Gazzotti, R.M. 1997. Effect of exogenous reduced glutathione on the survival of red blood cells in hemodialyzed patients. J Nephrol 10(5): 261-265.

Vashistha, T., Streja, E., Molnar, M.Z., Rhee, C.M., Moradi, H., Soohoo, M. et al. 2016. Red Cell Distribution Width and Mortality in Hemodialysis Patients. Am J Kidney Dis 68(1): 110-121. doi: 10.1053/j.ajkd.2015.11.020S0272-6386(15)01494-8 [pii].

Vilar, E., Fry, A.C., Wellsted, D., Tattersall, J.E., Greenwood, R.N., and Farrington, K. 2009. Longterm outcomes in online hemodiafiltration and high-flux hemodialysis: a comparative analysis. Clin J Am Soc Nephrol 4(12): 1944-1953. doi: 10.2215/CJN.05560809CJN.05560809 [pii].

Vos, F.E., Schollum, J.B., Coulter, C.V., Doyle, T.C., Duffull, S.B., and Walker, R.J. 2011. Red blood cell survival in long-term dialysis patients. Am J Kidney Dis 58(4): 591-598. doi: 10.1053/j.ajkd.2011.03.031S0272-6386(11)00849-3 [pii].

Vota, D.M., Maltaneri, R.E., Wenker, S.D., Nesse, A.B., and Vittori, D.C. 2013. Differential erythropoietin action upon cells induced to eryptosis by different agents. Cell Biochem Biophys 65(2): 145-157. doi: 10.1007/s12013-012-9408-4.

Weinstein, T., Chagnac, A., Korzets, A., Boaz, M., Ori, Y., Herman, M. et al. 2000. Haemolysis in haemodialysis patients: evidence for impaired defence mechanisms against oxidative stress. Nephrol Dial Transplant 15(6): 883-887. 
Wiecek, A., and Piecha, G. 2015. Is haemodiafiltration more favourable than haemodialysis for treatment of renal anaemia? Nephrol Dial Transplant 30(4): 523-525. doi: 10.1093/ndt/gfv029gfv029 [pii].

Yang, C.C., Hsu, S.P., Wu, M.S., Hsu, S.M., and Chien, C.T. 2006. Effects of vitamin C infusion and vitamin E-coated membrane on hemodialysis-induced oxidative stress. Kidney Int 69(4): 706-714. doi: S0085-2538(15)51535-4 [pii]10.1038/sj.ki.5000109. 
Table 1. Demographic, therapy-associated and hematological characteristics of the patient cohorts

\begin{tabular}{|c|c|c|c|c|c|}
\hline & \multicolumn{2}{|c|}{$\begin{array}{l}\text { HDF patients } \\
\qquad(n=16)\end{array}$} & \multicolumn{2}{|c|}{$\begin{array}{l}\text { cHD patients } \\
\qquad(n=16)\end{array}$} & \multirow[t]{2}{*}{$\begin{array}{l}\text { Controls } \\
(n=12)\end{array}$} \\
\hline & pre-dialysis & post-dialysis & pre-dialysis & post-dialysis & \\
\hline \multicolumn{6}{|l|}{ Clinical parameters } \\
\hline Age (years) & \multicolumn{2}{|c|}{$64 \pm 11$} & \multicolumn{2}{|c|}{$72 \pm 7$} & $60 \pm 14$ \\
\hline Weight (kg) & \multicolumn{2}{|c|}{$75 \pm 17$} & \multicolumn{2}{|c|}{$65 \pm 15$} & $60 \pm 10$ \\
\hline \multicolumn{6}{|l|}{ HD treatment } \\
\hline (months) & \multicolumn{2}{|c|}{$35 \pm 15$} & \multicolumn{2}{|c|}{$40 \pm 30$} & - \\
\hline Epo dose (IU/week) & \multicolumn{2}{|c|}{$9,100 \pm 5,527$} & \multicolumn{2}{|c|}{$9,350 \pm 4,204$} & - \\
\hline \multicolumn{6}{|l|}{ Biochemical } \\
\hline \multicolumn{6}{|l|}{ parameters } \\
\hline WBC (x10 $3 / \mu \mathrm{L})$ & $6.6 \pm 1.5$ & $6.5 \pm 1.2$ & $5.7 \pm 1.7$ & $5.8 \pm 2.3$ & $6.8 \pm 1.1$ \\
\hline $\mathrm{RBC}\left(\times 10^{6} / \mu \mathrm{L}\right)$ & $3.59 \pm 0.18$ & $4.10 \pm 0.35^{\dagger}$ & $3.61 \pm 0.40$ & $3.99 \pm 0.89$ & $4.77 \pm 0.56$ \\
\hline $\mathrm{Hb}(\mathrm{gr} / \mathrm{dL})$ & $11.7 \pm 0.3^{*}$ & $12.8 \pm 1.6^{\dagger}$ & $10.9 \pm 0.7^{*}$ & $11.8 \pm 1.0$ & $14.2 \pm 1.4$ \\
\hline Hct (\%) & $36.1 \pm 1.0^{*}$ & $37.9 \pm 4.2^{*}$ & $33.9 \pm 1.9^{*}$ & $33.2 \pm 3.8^{*}$ & $43.1 \pm 4.6$ \\
\hline $\operatorname{MCV}(f L)$ & $100.4 \pm 3.0$ & $93.0 \pm 6.0^{*, \dagger}$ & $95.4 \pm 9.0$ & $84.0 \pm 9.0^{*, \dagger}$ & $93.2 \pm 5.4$ \\
\hline $\mathrm{MCH}(\mathrm{pg})$ & $32.6 \pm 1.3^{*}$ & $31.7 \pm 2.0$ & $30.2 \pm 3.2^{*}$ & $29.6 \pm 4.4$ & $32.5 \pm 2.2$ \\
\hline $\mathrm{MCHC}(\mathrm{g} / \mathrm{dL})$ & $32.5 \pm 0.6^{*}$ & $34.1 \pm 1.3^{\dagger}$ & $31.2 \pm 0.6^{*}$ & $33.6 \pm 3.7$ & $33.7 \pm 1.5$ \\
\hline RDW (\%) & $15.1 \pm 1.1^{*}$ & $14.1 \pm 1.7$ & $16.6 \pm 1.4^{*}$ & $15.7 \pm 1.8$ & $12.8 \pm 0.9$ \\
\hline PTH (pg/mL) & $195 \pm 65$ & $N / D$ & $235 \pm 129$ & $N / D$ & $44 \pm 18$ \\
\hline Urea (mg/dL) & $143 \pm 23$ & $32 \pm 11^{\dagger}$ & $123 \pm 27$ & $38 \pm 9^{\dagger}$ & $31 \pm 12$ \\
\hline URR (\%) & \multicolumn{2}{|c|}{$78 \pm 6^{*}$} & \multicolumn{2}{|c|}{$69 \pm 7^{*}$} & - \\
\hline Creatinine (mg/dL) & $8.6 \pm 1.2^{*}$ & $N / D$ & $6.7 \pm 2.0^{*}$ & $N / D$ & $0.8 \pm 0.2$ \\
\hline Uric acid (mg/dL) & $7.9 \pm 1.8^{*}$ & $N / D$ & $6.1 \pm 1.0^{*}$ & $N / D$ & $4.2 \pm 1.2$ \\
\hline Cholesterol (mg/dL) & $148 \pm 29$ & $N / D$ & $145 \pm 36$ & $N / D$ & $145 \pm 18$ \\
\hline Triglycerides (mg/dL) & $150 \pm 60$ & $N / D$ & $146 \pm 42$ & $N / D$ & $132 \pm 29$ \\
\hline $\mathrm{HDL}(\mathrm{mg} / \mathrm{dL})$ & $45 \pm 11$ & $N / D$ & $43 \pm 11$ & $N / D$ & $73 \pm 9$ \\
\hline Calcium (mg/dL) & $9.3 \pm 0.2$ & $N / D$ & $9.3 \pm 0.3$ & $N / D$ & $9.3 \pm 0.2$ \\
\hline Phosphorus (mg/dL) & $5.0 \pm 0.6$ & $N / D$ & $4.8 \pm 0.8$ & $N / D$ & $3.6 \pm 0.6$ \\
\hline Potassium (mmol/L) & $5.0 \pm 0.3$ & $4.5 \pm 0.5$ & $4.9 \pm 0.5$ & $4.7 \pm 0.6$ & $4.2 \pm 0.3$ \\
\hline Sodium (mmol/L) & $137 \pm 2$ & $N / D$ & $137 \pm 2$ & N/D & $139 \pm 2$ \\
\hline $\mathrm{Fe}(\mu \mathrm{g} / \mathrm{dL})$ & $72.4 \pm 17.4$ & $N / D$ & $66.5 \pm 11.5$ & $N / D$ & $108.9 \pm 15.4$ \\
\hline Ferritin (ng/mL) & $914 \pm 436$ & $N / D$ & $831 \pm 487$ & $N / D$ & $72 \pm 18$ \\
\hline TIBC ( $\mu \mathrm{g} / \mathrm{dL})$ & $280 \pm 37^{*}$ & $N / D$ & $242 \pm 41^{*}$ & $N / D$ & $348 \pm 27$ \\
\hline Total proteins $(\mathrm{g} / \mathrm{dL})$ & $7.1 \pm 0.3$ & $N / D$ & $7.1 \pm 0.3$ & $N / D$ & $7.4 \pm 0.5$ \\
\hline Albumin (g/dL) & $4.3 \pm 0.3$ & $N / D$ & $4.3 \pm 0.6$ & $N / D$ & $4.2 \pm 0.4$ \\
\hline ALP (IU/L) & $83 \pm 19$ & $N / D$ & $79 \pm 12$ & $N / D$ & $63 \pm 10$ \\
\hline
\end{tabular}




\begin{tabular}{|c|c|c|c|c|c|}
\hline үGT (IU/L) & $17 \pm 7$ & $N / D$ & $14 \pm 5$ & $N / D$ & $12 \pm 9$ \\
\hline $\operatorname{TAC}\left(\mu \mathrm{M} \mathrm{Fe} e^{2+}\right)$ & $1,127 \pm 168$ & $401 \pm 65^{\dagger}$ & $1,021 \pm 198$ & $465 \pm 77^{\dagger}$ & $706 \pm 159$ \\
\hline UAiAC $\left(\mu \mathrm{M} \mathrm{Fe}^{2+}\right)$ & $449 \pm 90$ & $253 \pm 32^{\dagger}$ & $450 \pm 102$ & $261 \pm 53^{\dagger}$ & $239 \pm 45$ \\
\hline UAdAC $\left(\mu \mathrm{M} \mathrm{Fe} e^{2+}\right)$ & $678 \pm 104^{*}$ & $147 \pm 47^{*, \dagger}$ & $571 \pm 107^{*}$ & $199 \pm 45^{* \dagger}$ & $467 \pm 126$ \\
\hline
\end{tabular}

Values are presented as mean \pm SD. (N/D): not determined; bold, HD or HDF vs healthy subjects; ${ }^{*}$,

HDF vs HD; ${ }^{\dagger}$, pre- vs post- dialysis; $P<0.05$. ALP, alkaline phosphatase; $\gamma G T$, gamma-glutamyl transferase; PTH, parathormone; TAC, total antioxidant capacity; TIBC, total iron-binding capacity; UAdAC, uric acid-dependent antioxidant capacity; UAiAC, uric acid-independent antioxidant capacity; URR, urea reduction ratio.

Parameter (conversion factor) SI unit: White blood cell count (1.0) 109/L; Red blood cell count (1.0) 1012/L; Hemoglobin (10.0) g/L; Hematocrit (0.01) proportion of of 1.0; $\mathrm{MCH}(0.620) \mathrm{fmol}$; MCHC (0.620) mmol/L; PTH (1.0) ng/L; Urea (6.006) mmol/L; Creatinine (88.4) $\mu \mathrm{mol} / \mathrm{L}$; Uric acid (59.84) $\mu \mathrm{mol} / \mathrm{L}$; Cholesterol (0.0259) mmol/L; Triglycerides (0.0113) mmol/L; HDL (0.0259) mmol/L; Calcium (0.25) mmol/L; Phosphorus (0.323) mmol/L; Fe (0.179) $\mu \mathrm{mol} / \mathrm{L} ;$ Ferritin (2.247) pmol/L; TIBC (0.179) $\mu \mathrm{mol} / \mathrm{L}$; Total proteins (10.0) g/L; Albumin (10.0) g/L); ALP (16.66) $\mathrm{nmol} /(\mathrm{s} \cdot \mathrm{L}) ;$ jGT (16.66) $\mathrm{nmol} /(\mathrm{s} \cdot \mathrm{L})$. 


\section{Figure captions}

Fig. 1. Variation in RBC and plasma characteristics. Percentage of $\mathrm{PS}^{+} \mathrm{RBCs}\left(\mathrm{A} ;{ }^{* *}, P=0.026\right.$, and ${ }^{\# \text {, }}$ $P=0.0005$ ), levels of plasma free $\mathrm{Hb}$ (intravascular hemolysis, $\mathrm{B}{ }^{* *}, P=0.008$ ), intracellular $\mathrm{ROS}(\mathrm{C})$ and tBHP-induced intracellular ROS (D), before (pre) and after (post) dialysis session. HDF, cHD: patients under HDF or cHD treatment, respectively C: healthy subjects (controls). RFU: Relative fluorescence units. ${ }^{*}, P<0.05$ vs controls; ${ }^{* *}, P<0.05$ HDF vs $\mathrm{CHD} ;{ }^{\#}, P<0.05$ pre- vs post- dialysis session. Bars: Mean \pm SEM.

Fig. 2. RBC morphology and membrane exovesiculation in ESRD. (A) Box plots showing variation in stomatocytosis in HDF and CHD patients compared to healthy controls $\left(^{* *}, P=0.023\right)$. (B) Representative scanning electron micrographs of RBCs from patients under HDF or cHD treatment, after the dialysis session. Insert: typical stomatocytic shape transformations. Scale bars=10 $\mu$ m. (C) Flow cytometry analysis of total (MVs) and RBC-derived (R-MVs) microvesicles in the uremic plasma collected before $\left(^{* *}, P=0.010\right.$ for $\mathrm{MVs}$ and $P=0.049$ for $\mathrm{R}-\mathrm{MVs}$ ) and after dialysis, following normalization to healthy plasma $\left(100 \%\right.$, dotted line). Bars: Mean \pm SEM. ${ }^{*}, P<0.05$ vs control; ${ }^{* *}$, $P<0.05$ HDF vs $\mathrm{CHD} ;, \quad P<0.05$ pre- vs post- dialysis session.

Fig. 3. Variation in the protein composition of the RBC membrane in ESRD patients, before (A) and after (B) the dialysis session, following normalization to control (100\%, dotted line). Bars: Mean \pm SEM. ${ }^{*}, P<0.05$ vs control; ${ }^{* *}, P<0.05$ HDF vs $\mathrm{CHD;}{ }^{\#}, P<0.05$ pre- vs post- dialysis session. oxHb: oxidized/denatured $\mathrm{Hb}$. Insert images: representative immunoblots showing increased oxidized/denatured $\mathrm{Hb}$ species in HDF-RBC membrane isolated immediately post-dialysis. 


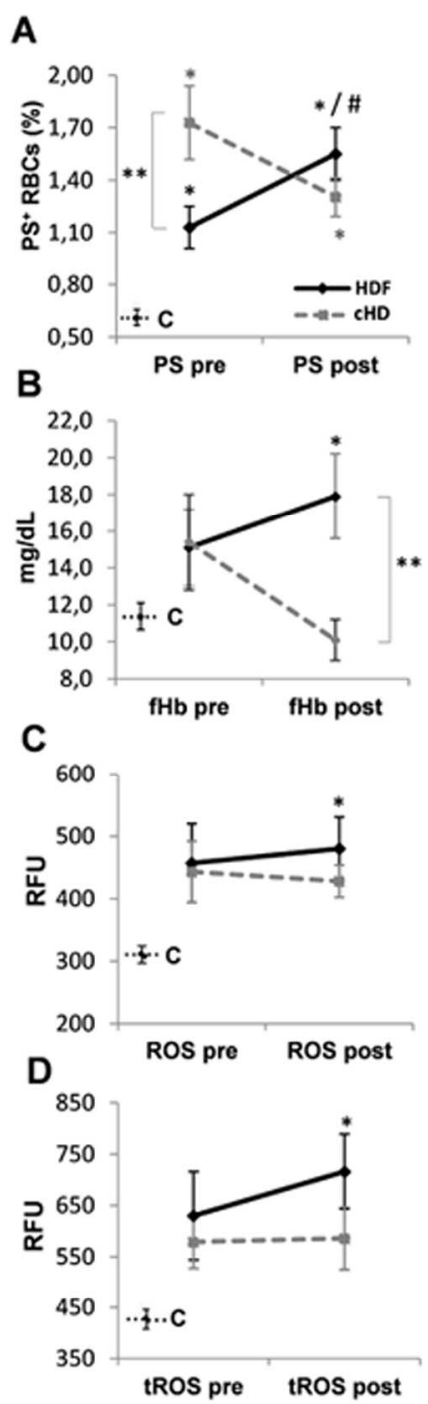

FIGURE 1

Fig. 1. Variation in RBC and plasma characteristics. Percentage of $P S+R B C s(A ; * *, P=0.026$, and \#, $\mathrm{P}=0.0005$ ), levels of plasma free $\mathrm{Hb}$ (intravascular hemolysis, $\mathrm{B} ; * *, \mathrm{P}=0.008$ ), intracellular $\mathrm{ROS}(\mathrm{C})$ and tBHP-induced intracellular ROS (D), before (pre) and after (post) dialysis session. HDF, CHD: patients under HDF or cHD treatment, respectively C: healthy subjects (controls). RFU: Relative fluorescence units. *, $\mathrm{P}<0.05$ vs controls; $* *, \mathrm{P}<0.05$ HDF vs $\mathrm{CHD} ; \#, \mathrm{P}<0.05$ pre- vs post- dialysis session. Bars: Mean $\pm \mathrm{SEM}$.

$197 \times 453 \mathrm{~mm}(600 \times 600 \mathrm{DPI})$ 
A

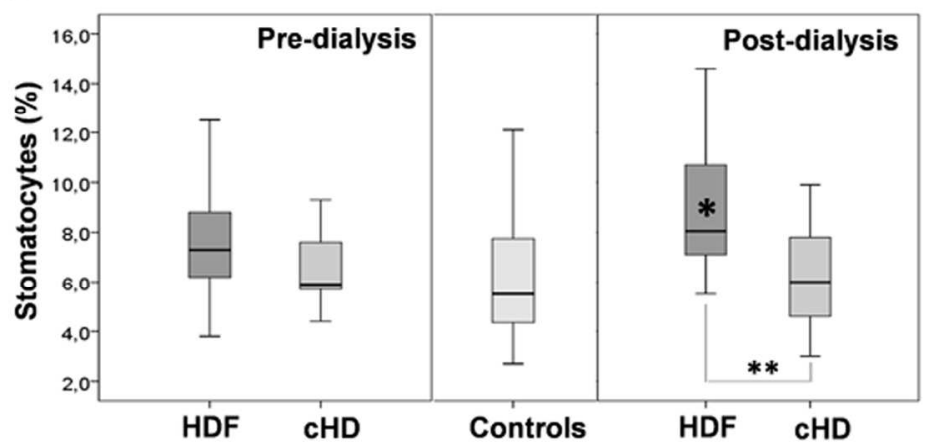

B
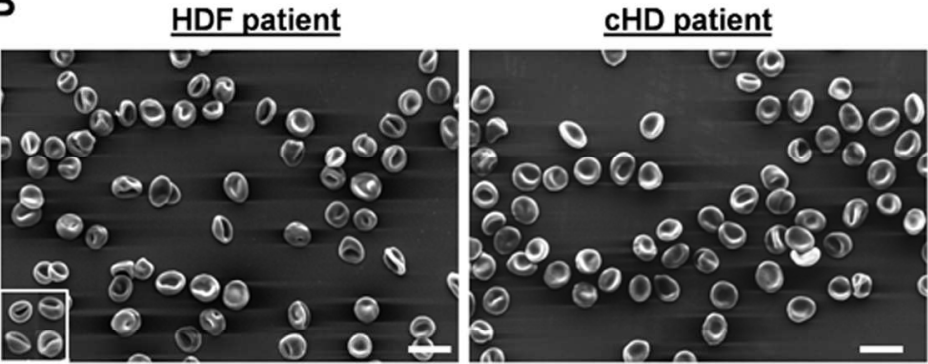

C

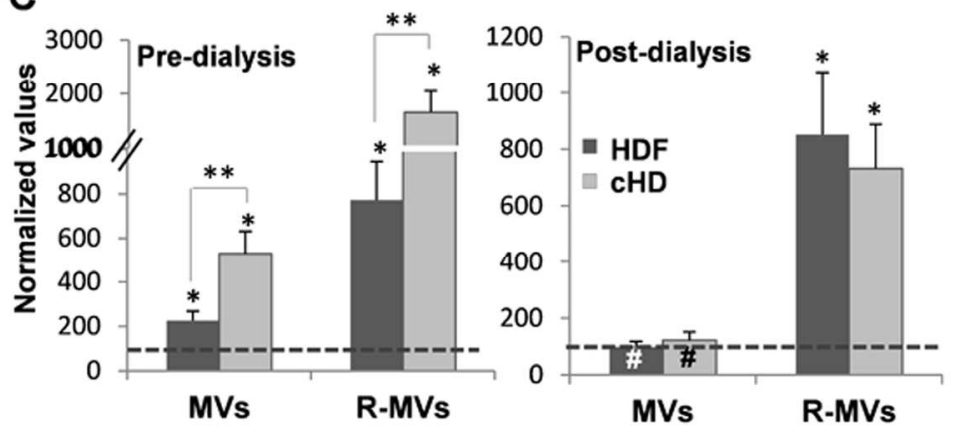

FIGURE 2

Fig. 2. RBC morphology and membrane exovesiculation in ESRD. (A) Box plots showing variation in stomatocytosis in HDF and CHD patients compared to healthy controls ( $* *, P=0.023)$. (B) Representative scanning electron micrographs of RBCs from patients under HDF or cHD treatment, after the dialysis session. Insert: typical stomatocytic shape transformations. Scale bars $=10 \mu \mathrm{m}$. (C) Flow cytometry analysis of total (MVs) and RBC-derived (R-MVs) microvesicles in the uremic plasma collected before $(* *, \mathrm{P}=0.010$ for MVs and $\mathrm{P}=0.049$ for R-MVs) and after dialysis, following normalization to healthy plasma ( $100 \%$, dotted line). Bars: Mean \pm SEM. *, $\mathrm{P}<0.05$ vs control; **, $\mathrm{P}<0.05$ HDF vs CHD; \#, $\mathrm{P}<0.05$ pre- vs post- dialysis session. 
A

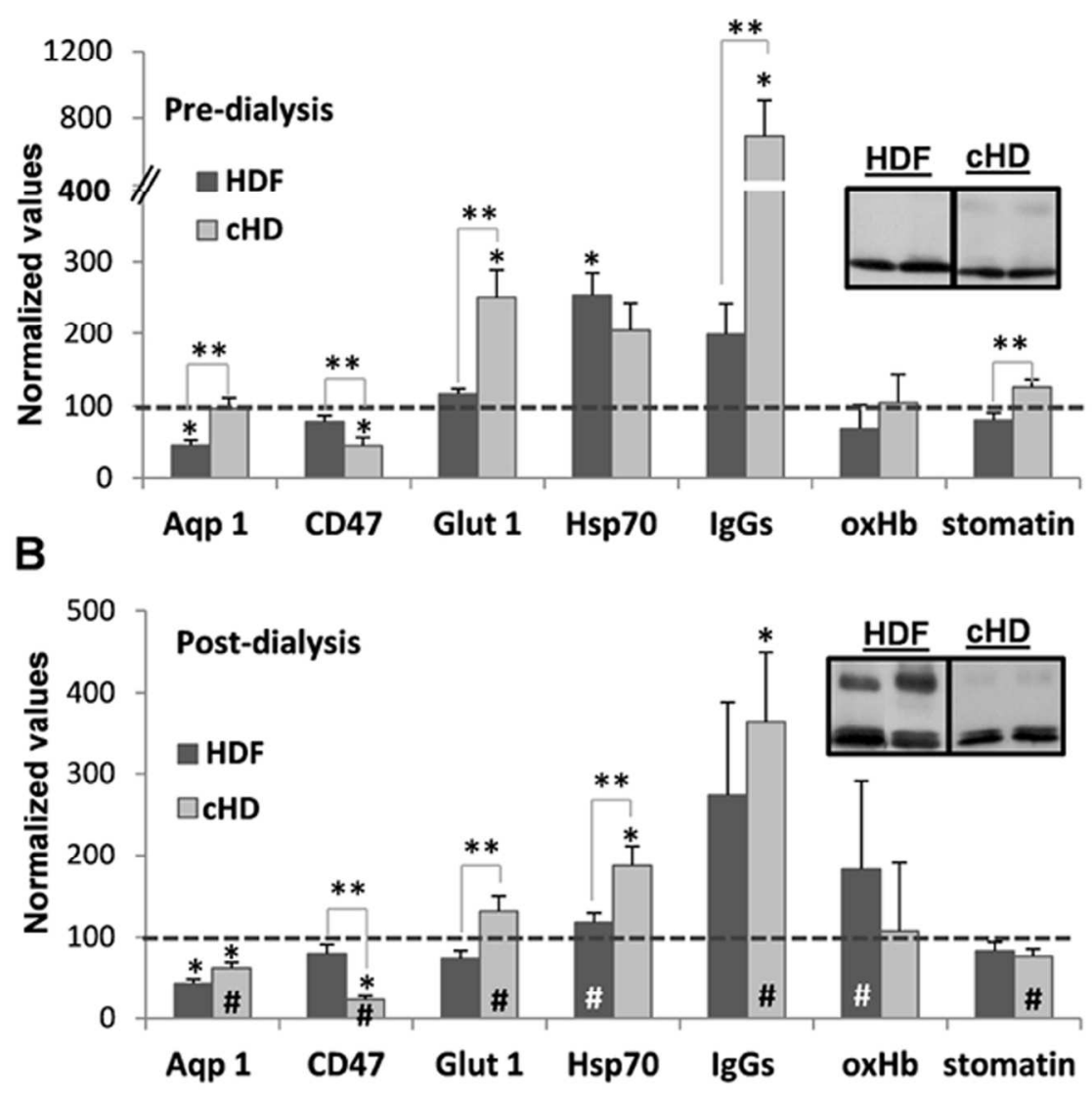

FIGURE 3

Fig. 3. Variation in the protein composition of the RBC membrane in ESRD patients, before (A) and after (B) the dialysis session, following normalization to control (100\%, dotted line). Bars: Mean \pm SEM. $*$, P<0.05 vs control; **, $\mathrm{P}<0.05$ HDF vs CHD; \#, $\mathrm{P}<0.05$ pre- vs post- dialysis session. oxHb: oxidized/denatured $\mathrm{Hb}$. Insert images: representative immunoblots showing increased oxidized/denatured Hb species in HDF-RBC membrane isolated immediately post-dialysis.

$208 \times 239 \mathrm{~mm}(300 \times 300 \mathrm{DPI})$ 